\title{
Lamina Slope Angle Is A Risk Factor for Ligamentum Flavum Hypertrophy in Patients with Lumbar Degenerative Disease: A Retrospective Study.
}

\author{
Xiaosheng Yu ( $\nabla$ johnsonyxs@hotmail.com ) \\ Shanghai Jiao Tong University School of Medicine Affiliated Renji Hospital \\ Junduo Zhao \\ Shanghai Jiao Tong University School of Medicine \\ Fan Feng \\ Shanghai Jiao Tong University School of Medicine Affiliated Renji Hospital \\ Yingchao Han \\ Shanghai Jiao Tong University School of Medicine Affiliated Renji Hospital \\ Guibin Zhong \\ Shanghai Jiao Tong University School of Medicine Affiliated Renji Hospital

\section{Zude Liu} \\ Shanghai Jiao Tong University School of Medicine Affiliated Renji Hospital \\ Jianwei Chen ( $\sim$ jwchenbone@126.com )
}

\section{Research article}

Keywords: laminar slope angle, lumbar disc degenerative grade, cross section area of multifidus, muscle-fat index, hypertrophy of ligamentum flavum

Posted Date: May 12th, 2020

DOI: https://doi.org/10.21203/rs.3.rs-27537/v1

License: (c) (i) This work is licensed under a Creative Commons Attribution 4.0 International License. Read Full License 


\section{Abstract}

Background.One previous work confirmed that laminar slope angle was associated with the ossification of thoracic ligamentum flavum. Several studies have investigated the relevance of disc degeneration grade, thickness of ligamentum flavum, along with cross section area of multifidus and its fatty infiltration. However, there has been no research between the angle and them. This retrospectivestudyof clinical materials from 122 patients was designed to investigate the influence of laminar slope angle on degeneration of lumbar when eliminating the interference of age.

Methods.122 individuals were retrospected randomly on the basis of age difference from 687inpatients scheduled to undergo a lumbar operation between January and December 2017.We registered their age and sex, evaluated and measuredL4-L5 disc degeneration grade,correspondingcross section area of multifidus, muscle-fat index,thickness of ligamentum flavum and laminar slope angle from preoperative magnetic resonance imagingand three-dimensional computer tomography. Independent-sample $T$ tests were used to assess the association between age and measurement indices. The Pearson correlation coefficient, and partial correlation excluding age, was also performed to analyze thecorrelation between clinical parameters.

Results.Our results showed that age was positively connected withthe laminar slope angle(L4:r=0.298, $p=\llbracket 0.01 ; L 5: r=0.303, p=\llbracket 0.01)$. Excluding the interference of age, revealed a credibly negative relationship between the angle of $L 4$ and the thickness of the ligamentum

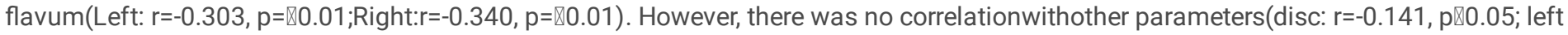
multifidus: $r=0.248$, $p \otimes 0.05$; right multifidus: $r=0.225$, $p \otimes 0.05$; left muscle-fat index: $r=0.033$, $p \otimes 0.05$; right muscle-fat index: $r=0.016, p \otimes 0.05$ ).

Conclusion.Excluding the interference of age, Inclination of small laminar slope angle leads to hypertrophy of lumbar ligamentum flavum.

\section{LEVEL OF EVIDENCE:Level 4.}

\section{Introduction}

Low back pain (LBP) is highly prevalent across the world, affects all ages, and is a the most common reason for patients living with disability ${ }^{1-4}$. A previous epidemiological investigation showed that the mean point prevalence was $18.3 \%^{5}$. LBP is common in people aged 60-65 years which cause by lumbar degenerative disease and can become more serious with age as the accumulation of mechanical stress damage increases ${ }^{6}$. Lumbar degeneration arises from a range of pathoanatomical alterations caused by mechanical stress, including lumbar disc degeneration(LDD), facet joint osteoarthritis(FJOA), hypertrophic ligamentum flavum(HLF), and paravertebral muscle atrophy. ${ }^{7}$

The spine bears the weight of our body under gravity. The functional spine unit (FSU) is a basic building block of the spine and is used to investigate the physical properties and functional biomechanics of the spine ${ }^{8}$. The FSU is composed of two adjacent vertebrae, the intervertebral disc, the facet joints, and the spinal ligaments. Many researchers have investigated the relative relationships between the components of the FSU as these can be measured or calculated easily. Pfirrmann et al. developed a formal classification scheme for discs in 2001 and allowed us to quantify and compare the degeneration of lumbar discs ${ }^{9}$. From then on, an increasing number of researchers and clinicians have investigated the relationship between lumbar disc degenerative grade and the degeneration of other FSU structures ${ }^{10-14}$.

In 2017, Kalichman et al. described three signs of muscle degeneration on imaging: decreased muscle size, decreased radiographic density, and the increased deposition of fat ${ }^{15}$. Sun et al. further reported that disc degeneration was positively correlated with the atrophy of the multifidus muscles at the L3-L4 disc level and emphasized strengthening the paraspinal muscle helps to prevent muscle atrophy and slowed down the progression of lumbar spinal degeneration ${ }^{11}$.

The laminar slope angle(LSA) was first proposed by Xu in 1999and defined as the intersection angle of the plane of the lamina and the horizontal plane of the vertebral body ${ }^{16}$, thus representing the tilt of each lamina. This angle did represent an anatomical index that reflected the relative tilt of the lamina and was first used to provide a preference for spinal surgeons to place sublaminar instruments. Qin's work confirmed that the LSA was associated with the ossification of the ligamentum flavum in thoracic segments but there was no research about the relationship between LSA and lumbar structure ${ }^{17}$. Thus, we hypothesize that the extent of the LSA may affect the vertebral anatomical structure and give rise to LDD, HLF, multifidus muscle atrophy and fatty infiltration. These changes could lead to degenerative lumbar disease.

\section{Materials And Methods}




\section{Study participants}

We retrospected a total of 687 patients, of all ages, who came to the inpatient department of spinal surgery at Renji hospital and were scheduled for lumbar surgeries between January and December 2017. We divided these patients into five groups according to age: below 35 years, between 36 and 45 years, between 46 and 55 years, between 56 and 65 years, and above 66 years. Then, we randomly selected 20-30 samples(a ratio of 6:1) from each group and mixed them up to create a final sample group. A total of 122 patients remained in the analysis (Fig. 1). We registered the age and sex of all 122 patients, who all underwent magnetic resonance imaging(MRI) and computerized tomography(CT) scans of the lumbar spine prior to surgery. The inclusion criteria were: (1) no history of spinal surgery; (2) no recent history of severe lumbar trauma; (3) no abnormal radiological findings, such as vertebral fractures, space occupying lesions of the lumbar spine, or apparent spinal deformities (e.g., scoliosis); and (4) no history of systemic diseases (rheumatic diseases of the spine or carcinoma).

The study protocol was approved by the ethics committee of Renji Hospital, School of Medicine, Shanghai Jiao Tong University. Informed consent was were obtained from each patient prior to the imaging examination.

\section{Magnetic Resonance Imaging Protocol}

As reported previously by $\mathrm{Yu}^{10}$, all T2-weighted images were acquired using the same 3.0 T imaging system (Magnetom; Siemens, Erlanger, Germany) with a repetition time of $3220 \mathrm{~ms}$ and an echo time of $120 \mathrm{~ms}$. Slice thickness was $4 \mathrm{~mm}$. The acquisition matrix was $512 \times 512$ and the field of view was $310 \mathrm{~mm}$. We then obtained and evaluated Original Digital Imaging and Communications in Medicine files from transverse oblique MRI images parallel to the superior end plate of L4 and L5.

\section{Computed Tomography Protocol}

Preoperative patients who were eligible for CT underwent imaging with an 8-slice multidetector CT scanner (Lightspeed Ultra; GE, Milwaukee, Wisconsin). Each patient underwent unenhanced lumbar CT performed with a sequential scan protocol with slice collimation of $8 \times 2.5 \mathrm{~mm}(120 \mathrm{kVp}, 320 / 400 \mathrm{~mA}$ for $0.220 \mathrm{lb}$ body weight) during a single end-inspiratory breath hold (typical duration, $18 \mathrm{~s})$. For the lumbar scan, 256 contiguous $2.5 \mathrm{~mm}$ slices of the lumbar region were acquired, covering a $150 \mathrm{~mm}$ area above the level of S1. The evaluation of CT scans was performed with blinding to clinical and personal data.

\section{Disc Degeneration Assessment}

Disc degeneration assessment used the disc degeneration grade described by Pfirrmann in $2001^{9}$. Observers analyzed the L4-L5 lumbar intervertebral disc from each patient on T2-weight sagittal MRI images using Picture Archiving and Communication Systems (PACS), version 11.4 (Carestream health, Shanghai, China)(Fig. 2) with Pfirrmann's original article to confirm the grade at the time of evaluation. Independently, more than half of the selected grade was recorded. If there was a dispute, then images were reevaluated until more than half of the observers agreed.

\section{Laminar Slope Angle Measurements}

Laminar slope angle was measured with PACS. We reconstructed pre-operation CT in three dimensions and proofread the central axis in parallel with the direction of the spinous process on the axis CT image and with the postural tilt of the spine on the coronal image. We then chose the sagittal images at the level of the tip of the unilateral facet joints (Fig. 3). Evaluators analyzed the selected sagittal CT images using our redefined method similar to Bai-ling's on lateral radiographs ${ }^{18}$ We drew two separate lines connecting the tip of the superior facet with the base of the inferior facet and connecting the midpoints of the anterior and posterior vertebral cortices; we then measured the intersecting angle between the two lines. We selected L4 and L5 axis position on sagittal CT images, measured the corresponding LSA, and calculated the absolute value of the difference between the two adjacent segments of LSA (Fig. 2)

\section{Muscle Measurements And Analysis}

Muscle measurements and analysis were carried out with PACS and Image $\mathrm{J}$ software, version 1.42q (National Institutes of Health, Bethesda, Maryland) following the method described in our previous article ${ }^{10}$. We selected the axial slice at the level of the L 5 vertebral body upper endplate to calculate L4-5 muscle cross section area(CSA) (Fig. 2). Intramuscular fatty infiltration was obtained with a widely 
accepted muscle-fat index, which represents the ratio of mean signal intensity in a region of lean muscle tissue relative to the signal intensity in a homogeneous region of fat (Fig. 2).

\section{Thickness Of The Ligamentum Flavum}

The thickness of the ligamentum flavum was measured on axis T2-weight MRI images with PACS. We located the spinal level of the L4-5 intervertebral spaces on sagittal T2-weight MRI images and selected the axial slice at the level of the L5 vertebral body upper endplate. ${ }^{13,19-21}$ We then drew two parallel lines along the direction of the ligamentum flavum and chose the maximum distance between the dural side and the dorsal side. The maximum thickness of the LF was measured on both the right and left sides (Fig. 2)

\section{Reliability Tests}

To avoid bias, two radiologists and two surgeons were blinded to the study design; consequently, when measuring the parameters, they all ignored the laminar slope angle. To ensure the objectivity of the results, all measurements were repeated by the radiologists and surgeons 2 weeks after the initial evaluation. The mean of the data was then used in the primary analysis.

\section{Statistical Analysis}

Statistical analyses were performed with SPSS software, version 24.0 (IBM, Armonk, New York). The association between age and measurement indexes were determined by the independent-samples $T$ test. The association between laminar slope angle and other parameters were determined by Pearson correlation. Partial correlation was used to analyze the correlation of other remaining variables while controlling age variables. The reliability of the measured parameters was evaluated using intraclass correlation coefficients. Significance was set at $\mathrm{P}<0.05$ and values represent mean \pm standard error.

\section{Results}

A total of 122 patients were in our study including 66 men and 56 women (1.18:1). The mean age was $50.97 \pm 14.78$ years, ranging from 22 to 86 years. The descriptive anthropometric characteristics of the patients are shown in Table 1. The Cronbach's alpha was 0.67 ; thus, the quantity of all measurements had good credibility. The independent-samples T-test showed that males were younger than the females. Women also had a smaller multifidus and more serious fatty infiltration, but there was no significant difference in LSA and ligamentum flavum when compared between the male and female group (Table 2).

Table 1

Patient Demographics Characteristics

\begin{tabular}{|llll|}
\hline \multicolumn{4}{|c|}{ Patient Demographics Characteristics } \\
\hline Characteristics & Number & No.of patients(\%) & $\begin{array}{l}\text { Value } \\
\text { (range) }\end{array}$ \\
\hline Male & & & - \\
\hline Female & 66 & 54.1 & \\
\hline Age,yr & 56 & 45.9 & \\
\hline$\square 35$ & & & \\
\hline $36-45$ & 19 & 15.6 & \\
\hline $46-55$ & 28 & 23.0 & \\
\hline $56-65$ & 33 & 27.0 & \\
\hline$\square 66$ & 22 & 18.0 & \\
\hline Value = Mean \pm SD & 16.4 & \\
\hline
\end{tabular}


Table 2

Result of independent-samples T test

\begin{tabular}{|c|c|c|c|c|}
\hline & Male(n= 66) & Female(n = 56) & $\mathbf{t}$ & $\mathbf{P}$ \\
\hline Age & $48.29 \pm 15.43$ & $54.13 \pm 13.59$ & -2.20 & $0.03^{*}$ \\
\hline Disc grade & $3.15 \pm 0.83$ & $3.30 \pm 0.76$ & -1.05 & 0.30 \\
\hline \multicolumn{5}{|c|}{ CSA of muscle, $\left(\mathrm{mm}^{2}\right)$} \\
\hline Left & $751.71 \pm 172.20$ & $613.61 \pm 126.12$ & 3.89 & $\nabla 0.01^{\star *}$ \\
\hline Right & $731.67 \pm 159.31$ & $603.10 \pm 146.54$ & 3.65 & $\nabla 0.01^{\star \star}$ \\
\hline Total & $1483.39 \pm 312.27$ & $1216.71 \pm 261.51$ & 3.99 & $\nabla 0.01^{\star \star}$ \\
\hline \multicolumn{5}{|c|}{ Musle-fat index, (\%) } \\
\hline Left & $27.80 \pm 9.86$ & $34.10 \pm 9.71$ & -2.98 & $\nabla 0.01^{\star \star}$ \\
\hline Right & $30.08 \pm 11.89$ & $35.71 \pm 9.89$ & -2.37 & $0.02^{*}$ \\
\hline Total & $28.94 \pm 10.68$ & $34.91 \pm 9.55$ & -2.72 & $\nabla 0.01^{\star \star}$ \\
\hline LSA & $111.10 \pm 5.81$ & $110.27 \pm 6.27$ & 0.706 & 0.48 \\
\hline \multicolumn{5}{|c|}{ Thickness of LF, (mm) } \\
\hline Left & $2.90 \pm 0.98$ & $2.97 \pm 1.16$ & -0.366 & 0.72 \\
\hline Right & $2.90 \pm 0.94$ & $3.01 \pm 1.03$ & -0.576 & 0.57 \\
\hline \multicolumn{5}{|c|}{$\mathrm{CSA}=$ cross-sectional area; LSA = lamina slope angle; LF = ligamentum flavum. } \\
\hline
\end{tabular}

\section{Correlation Analysis}

Correlation analysis (Table 3) showed that age was a factor responsible for differences in the LSA (L4: $r=0.298, L 5: r=0.303)$. Also, age had significant correlations with CSA of the multifidus(Left: $r=-0.448$, Right: $r=-0.462)$, muscle-fat index (Left: $r=0.238$, Right: $r=0.266$ ), degeneration of the lumbar disc $(r=0.299)$ and thickness of ligamentum flavum(Left: $r=0.294$, Right: $r=0.283)$. L4-5 disc degeneration was positively associated with the fatty infiltration(Left: $r=0.301$, Right: $r=0.286$ ). There was no correlation between the LSA of L4 and the CSA of the multifidus while there was a negative relationship between the LSA of L5 and it (Left: $r=-0.375$, Right: $r=-0.352$ ). Differences in the LSA did not affect the degeneration of the lumbar disc. Only the thickness of the right L4-5 ligamentum flavum showed a negative relationship with the LSA of L4 ( $r=-0.227)$. 
Table 3

Result of Pearson of correlation coefficient

\begin{tabular}{|c|c|c|c|c|c|c|c|c|c|c|c|c|}
\hline & & \multirow[t]{2}{*}{ age } & \multirow{2}{*}{$\begin{array}{l}\text { Disc } \\
\text { grade }\end{array}$} & \multicolumn{2}{|c|}{ CSA of multifidus } & \multicolumn{2}{|c|}{ Muscle-fat index } & \multirow{2}{*}{$\begin{array}{l}\text { LSA of } \\
\text { L4 }\end{array}$} & \multirow{2}{*}{$\begin{array}{l}\text { LSA of } \\
\text { L5 }\end{array}$} & \multirow{2}{*}{$\begin{array}{l}\text { Difference } \\
\text { value of } \\
\text { LSA }\end{array}$} & \multicolumn{2}{|c|}{ Thickness of LF } \\
\hline & & & & Left & Right & Left & Right & & & & Left & Right \\
\hline \multicolumn{2}{|l|}{ age } & 1 & $0.299^{\star \star}$ & $-0.448^{\star \star}$ & $-0.462^{\star \star}$ & $0.238^{*}$ & $0.266^{*}$ & $0.298^{\star \star}$ & $0.303^{\star *}$ & 0.082 & $0.294^{\star \star}$ & $0.283^{\star \star}$ \\
\hline \multicolumn{2}{|l|}{ Disc grade } & - & 1 & -0.131 & -0.191 & $0.301^{*}$ & $0.286^{*}$ & -0.040 & -0.065 & 0.059 & -0.108 & 0.013 \\
\hline \multirow{2}{*}{$\begin{array}{l}\text { CSA of } \\
\text { multifidus }\end{array}$} & Left & - & - & 1 & $0.822^{\star \star}$ & -0.213 & -0.128 & -0.110 & $-0.375^{\star \star}$ & -0.095 & -0.059 & -0.099 \\
\hline & Right & - & - & - & 1 & $-0.281^{\star}$ & $-0.283^{\star}$ & -0.150 & $-0.352^{\star \star}$ & -0.007 & 0.033 & -0.050 \\
\hline \multirow{2}{*}{$\begin{array}{l}\text { Muscle- } \\
\text { fat index }\end{array}$} & Left & - & - & - & - & 1 & $0.918^{\star \star}$ & 0.102 & 0.172 & 0.104 & 0.055 & 0.068 \\
\hline & Right & - & - & - & - & - & 1 & 0.094 & $0.259^{*}$ & 0.124 & 0.147 & 0.134 \\
\hline \multicolumn{2}{|l|}{ LSA of L4 } & - & - & - & - & - & - & 1 & $0.433^{\star \star}$ & $-0.237^{*}$ & -0.189 & $-0.227^{*}$ \\
\hline \multicolumn{2}{|l|}{ LSA of L5 } & - & - & - & - & - & - & - & 1 & 0.191 & -0.093 & 0.103 \\
\hline \multicolumn{2}{|c|}{$\begin{array}{l}\text { Difference value } \\
\text { of LSA }\end{array}$} & - & - & - & - & - & - & - & - & 1 & 0.191 & 0.181 \\
\hline \multirow{2}{*}{$\begin{array}{l}\text { Thickness } \\
\text { of LF }\end{array}$} & Left & - & - & - & - & - & - & - & - & - & 1 & $0.892^{\star *}$ \\
\hline & Right & - & - & - & - & - & - & - & - & - & - & 1 \\
\hline *mean $\mathrm{P} \otimes 0.05,{ }^{* *}$ mean $\mathrm{P} \otimes 0.01$. & \multicolumn{12}{|c|}{ CSA = cross-sectional area; LSA = lamina slope angle; LF = ligamentum flavum; } \\
\hline
\end{tabular}

\section{Partial Correlation Analysis}

After controlling for age, our results (Table 4) revealed a credibly negative relationship between the LSA of L4 and the thickness of the ligamentum flavum(Left: $r=-0.303$, Right: $r=-0.340$ ), although there was no correlation with the CSA of the multifidus and fatty infiltration. There was no significant relationship between the LSA of L5 and any of these measurement indices. LSA did not affect degeneration of the lumbar disc. 
Table 4

Result of partial correlation coefficient

\begin{tabular}{|c|c|c|c|c|c|c|c|c|c|c|c|}
\hline & & \multirow{2}{*}{$\begin{array}{l}\text { Disc } \\
\text { grade }\end{array}$} & \multicolumn{2}{|l|}{ CSA } & \multicolumn{2}{|c|}{ Muscle-fat index } & \multirow{2}{*}{$\begin{array}{l}\text { LSA of } \\
\text { L4 }\end{array}$} & \multirow{2}{*}{$\begin{array}{l}\text { LSA of } \\
\text { L5 }\end{array}$} & \multirow{2}{*}{$\begin{array}{l}\text { Difference } \\
\text { value of LSA }\end{array}$} & \multicolumn{2}{|c|}{ Thickness of LF } \\
\hline & & & Left & Right & Left & Right & & & & Left & Right \\
\hline \multicolumn{2}{|l|}{ Disc grade } & 1 & 0.003 & -0.063 & 0.248 & 0.225 & -0.141 & -0.171 & 0.036 & -0.214 & -0.078 \\
\hline \multirow{2}{*}{$\begin{array}{l}\text { CSA of } \\
\text { multifidus }\end{array}$} & Left & - & 1 & $0.776^{* *}$ & -0.123 & -0.010 & 0.028 & -0.281 & -0.066 & 0.085 & 0.032 \\
\hline & Right & - & - & 1 & -0.198 & -0.187 & -0.015 & $-0.251^{*}$ & 0.051 & 0.199 & 0.095 \\
\hline \multirow{2}{*}{$\begin{array}{l}\text { Musclefat } \\
\text { index }\end{array}$} & Left & - & - & - & 1 & $0.913^{* *}$ & 0.033 & 0.108 & 0.087 & -0.016 & 0.001 \\
\hline & Right & - & - & - & - & 1 & 0.016 & 0.194 & 0.107 & 0.075 & 0.064 \\
\hline \multicolumn{2}{|l|}{ LSA of L4 } & - & - & - & - & - & 1 & -0.091 & -0.118 & $-0.303^{\star \star}$ & $-0.340^{\star \star}$ \\
\hline \multicolumn{2}{|l|}{ LSA of L5 } & - & - & - & - & - & - & 1 & 0.176 & 0.005 & 0.019 \\
\hline \multicolumn{2}{|c|}{$\begin{array}{l}\text { Difference value of } \\
\text { LSA }\end{array}$} & - & - & - & - & - & - & - & 1 & 0.175 & 0.165 \\
\hline \multirow{2}{*}{$\begin{array}{l}\text { Thickness of } \\
\text { LF }\end{array}$} & Left & - & - & - & - & - & - & - & - & 1 & $0.882^{\star \star}$ \\
\hline & Right & - & - & - & - & - & - & - & - & - & 1 \\
\hline
\end{tabular}

\section{Discussion}

LSA was varies across different vertebral levels: the maximum angle was $129.0 \pm 7.5^{\circ}$ at the L3 level while the minimum angle was $97.8 \pm$ $3.0^{\circ}$ at the T9 level ${ }^{16}$. Qin et al. further reported that the thoracic laminar slope angle had a negative relationship with tension acting on the ligamentum flavum and may also represent an anatomical and biomechanical factor in the lower thoracic spine that could be related to thoracic ossification of the ligamentum flavum ${ }^{17}$. Hypertrophy and ossification are both part of the degenerative progression in the ligament ${ }^{22}$. Thus, we considered whether the same phenomenon might existed in the lumbar region or whether other structures of the FSU are associated with the LSA. A diverse LSA may lead to different degenerative changes in these structures.

Our study was the first one to investigate the relationship between the laminar slope angle and lumbar degenerative disease. Through analysis we found that age was an important element which correlated to almost all of the measurement indices except for the difference value of the two segment LSA. The reduction in the CSA of the multifidus, the increased lumbar disc degeneration grade, and the area of fatty infiltration, with increasing age is consistent with existing research ${ }^{23-27}$. LSA was positively related to age, this can be explained by the osteoproliferation caused by FJOA. Age is known to be one of the risk factors of FJOA; the main group of patients with FJOA were those of advanced age. If FJOA occurs repeatedly, it could lead to osteoproliferation in the facet joints ${ }^{28}$.

Calculating the angle of the lamina slope, we drew one straight line connecting the tip of the superior facet and the base of inferior facet. As the facet joint protruded, the line lay flatter (as shown in Fig. 4); consequently, LSA was larger. This is the opposite of vertebral body compression. Compression changes in the vertebrae tend to be concentrated in the anterior column which is from the three column theory, proposed by Dennis in 1983, and modified by Allen in $1984^{29}$. With a reduction in anterior vertebrae height, the horizontal plane of the vertebral body leans forwards and downwards; this results in the LSA becoming smaller (Fig. 4).

The muscle-fat index is a ratio of signal intensity, it become larger represents more fatty infiltration. Our results showed that age and LDD was positively related to fatty infiltration; this concurred with Dahlqvist's opinion, who reported that the paraspinal muscles were more susceptible to fatty infiltration and age-related change ${ }^{30}$, and also Sun's conclusion ${ }^{11}$ in that we need more exercise to slow down the process of muscle disuse-atrophy and neurotic atrophy caused by LDD.

Meanwhile, to optimize our results we excluded the inference of age for partial correlation analysis. This analysis showed a credibly negative relationship between the LSA of L4 and thickness of ligamentum flavum. The ligamentum flavum, which is part of the posterior ligamentous complex, and maintains the stability of the posterior column of the spine ${ }^{21}$. This was located under the lamina and connected 
the adjacent two vertebrae. Our spine represents the axial bone which carries our upper body weight whenever it is stretched or remains still $^{31}$. Thus, when the lumbar bears the upper body weight, gravity can be decomposed into tension along the lamina and vertical lamina tension (Fig. 5).

The tension along the lamina could be referred to as an acting force and according to Newton's third law, the internal tension of the LF would be the reacting force equal to, and working against it. The internal tension of $L F$ can be described as $F_{L F}=G * C o s \beta$, where $G$ represents the upper body gravity, a represents LSA and $\beta \propto a\left(\right.$ Fig. 5). The angle sum of a triangle is 180 . Thus, in a right triangle $\beta \in\left[0,90^{\circ}\right]$ and the Cosine graph was a descending curve. Using this equation, $\beta$ was negatively related to $\operatorname{Cos} \beta$. $G$ was a constant quantity for an individual; so, a was also negatively related to $\mathrm{F}_{\mathrm{LF}}$. Therefore, the tension along the lamina was negatively correlated with LSA. Hayashi et al. previously reported that LF, with concentrated mechanical stress, showed degeneration with disruption of the elastic fibers and an increase in the cartilage matrix increase; this is similar to HLF from patients with lumbar spinal stenosis(LSS) ${ }^{32}$. When LSA was small, the mechanical stress is larger, and the corresponding LF was more likely to be hypertrophied. This explains why the thickness of the ligamentum flavum has a strong negative relationship with the LSA of L4. As Jun highlighted that a high T1 slope might be a predisposing factor in degenerative cervical spondylolisthesis ${ }^{33}$, while other research stated that the sacral slope was of importance because a reduction in the sagittal balance of the spine could cause chronic low back pain in patients with degenerative lumbar scoliosis ${ }^{34}$. We inferred that small LSA lead to HLF which was one reason of LSS. Smaller LSA may be one predisposing factor of LSS.

There are several limitations in our research that should be taken into consideration. First, this was a study based in a single center; our conclusions therefore need to be verified in future multi-center studies. Second, we did not consider the effect of body mass index, which can affect fatty infiltration, the CSA of the multifidus muscle, and cause stress on the ligament flavum. Osteoproliferation appeared to be associated with the LSA, our results did not consider the grade of osteoproliferation in the facet joints. Further research could explore the relationship between these factors.

\section{Conclusion}

LSA increases with age and therefore, provides a good index to reflect the morphological differences of the individual lumbar vertebrae. When excluding the influence of age, our results showed that LSA will not affect LDD, or the size and quality of the multifidus, but it will have a certain degree of influence on the HLF. The smaller the LSA, the thicker the corresponding segment of ligamentum flavum. It may increase the risk of hypertrophy of ligamentum flavum in future.

\section{List Of Abbreviations}

\begin{tabular}{|ll|}
\hline Abbreviations & Full name \\
\hline LBP & Low back pain \\
\hline LDD & lumbar disc degeneration \\
\hline FJOA & facet joint osteoarthritis \\
\hline HLF & hypertrophic ligamentum flavum \\
\hline FSU & functional spine unit \\
\hline LSA & laminar slope angle \\
\hline MRI & magnetic resonance imaging \\
\hline CT & computerized tomography \\
\hline PACS & Picture Archiving and Communication Systems \\
\hline CSA & cross section area \\
\hline LSS & lumbar spinal stenosis \\
\hline
\end{tabular}

\section{Declarations}

\section{Ethics approval and consent to participate}


The study protocol was approved by the ethics committee of Renji Hospital, School of Medicine, Shanghai Jiao Tong University. Informed consent was were obtained from each patient prior to the imaging examination.

\section{Consent for publication}

Not applicable.

\section{Availability of data and materials}

The datasets used and analysed during the current study are available from the corresponding author on reasonable request.

\section{Competing interests}

The authors declare that they have no competing interests.

\section{Funding}

This work was supported by Medical-Engineering Joint Fund of Shanghai Jiao Tong University (No. YG2015MS50).

\section{Authors' contributions}

XYhave made substantial contributions to the data acquisition, analysis and have drafted the work or substantively revised it. JZ have made substantial contributions to the data acquisitionand analysis. FF andYHhave made substantial contributions tointerpretation of data. GZ and ZLhave made substantial contributions to data provide as they are surgeon of all patients. JC have made substantial contributions to the conception OR design of the work.

\section{Acknowledgements}

Thanks for the support of imaging department of Renji Hospital, Shanghai Jiao Tong University School of Medicine.

\section{Authors' information (optional)}

XY,Department of Orthopaedic Surgery, Renji Hospital, Shanghai Jiao Tong University School of Medicine, 1630 Dongfang Road, Shanghai 200127, China.Email:johnsonyxs@hotmail.com

JC, Department of Orthopaedic Surgery, Renji Hospital, Shanghai Jiao Tong University School of Medicine, 1630 Dongfang Road, Shanghai 200127, China.Email: jwchenbone@126.com

\section{References}

1. Zaina F, Tomkins-Lane C, Carragee E, Negrini S. Surgical versus non-surgical treatment for lumbar spinal stenosis. Cochrane Database Syst Rev 2016; (1): CD010264.

2. Lurie J, Tomkins-Lane C. Management of lumbar spinal stenosis. BMJ. 2016;352:h6234.

3. Joseph JR, Farooqui Z, Smith BW, et al. Does clinical improvement of symptomatic degenerative lumbar disease impact obesity? J Neurosurg Spine. 2017;26(6):705-8.

4. Buser Z, Ortega B, D'Oro A, et al. Spine Degenerative Conditions and Their Treatments: National Trends in the United States of America. Global Spine J. 2018;8(1):57-67.

5. Maher C, Underwood M, Buchbinder R. Non-specific low back pain. The Lancet. 2017;389(10070):736-47.

6. Wang F, Cai F, Shi R, Wang XH, Wu XT. Aging and age related stresses: a senescence mechanism of intervertebral disc degeneration. Osteoarthritis Cartilage. 2016;24(3):398-408. 
7. Hansen BB, Hansen P, Carrino JA, Fournier G, Rasti Z, Boesen M. Imaging in mechanical back pain: Anything new? Best Pract Res Clin Rheumatol. 2016;30(4):766-85.

8. Oxland TR. Fundamental biomechanics of the spine-What we have learned in the past 25 years and future directions. $\mathrm{J}$ Biomech. 2016;49(6):817-32.

9. Pfirrmann CWM, Zanetti A, Hodler M, Boos J. N. Magnetic Resonance Classification of Lumbar Intervertebral Disc Degeneration. Spine (Phila Pa 1976). 2001;26(17):1873-8.

10. Yu B, Jiang K, Li X, Zhang J, Liu Z. Correlation of the Features of the Lumbar Multifidus Muscle With Facet Joint Osteoarthritis. Orthopedics. 2017;40(5):e793-800.

11. Sun D, Liu P, Cheng J, Ma Z, Liu J, Qin T. Correlation between intervertebral disc degeneration, paraspinal muscle atrophy, and lumbar facet joints degeneration in patients with lumbar disc herniation. BMC Musculoskelet Disord. 2017;18(1):167.

12. Sugawara K, Katayose M, Watanabe K. The Variation in the Lumbar Facet Joint Orientation in an Adult Asian Population and Its Relationship with the Cross-Sectional Area of the Multifidus and Erector Spinae. Asian Spine J. 2016;10(5):886-92.

13. Kolte VS, Khambatta S, Ambiye MV. Thickness of the ligamentum flavum: correlation with age and its asymmetry-an magnetic resonance imaging study. Asian Spine J. 2015;9(2):245-53.

14. Shahidi B, Parra CL, Berry DB, et al. Contribution of Lumbar Spine Pathology and Age to Paraspinal Muscle Size and Fatty Infiltration. Spine (Phila Pa 1976). 2017;42(8):616-23.

15. Kalichman L, Carmeli E, Been E. The Association between Imaging Parameters of the Paraspinal Muscles, Spinal Degeneration, and Low Back Pain. Biomed Res Int 2017; 2017: 2562957.

16. Xu RB, Ebraheim A, Yeasting NA. R. A. The Quantitative Anatomy of the Laminas of the Spine. Spine (Phila Pa 1976). 1999;24(2):10733.

17. Qin DZ, Li ZL, Cui XD, Liu XD. F. Anatomical significance of thoracic laminar slope angle on thoracic ossification of ligamentum flavum. CHINESE JOURNAL OF CLINICAL ANATOMY. 2006;24(6):634-6.

18. Chen BL, Wei FX, Ueyama K, Xie DH, Sannohe A, Liu SY. Adjacent segment degeneration after single-segment PLIF: the risk factor for degeneration and its impact on clinical outcomes. Eur Spine J. 2011;20(11):1946-50.

19. Sakamaki T, Sairyo K, Sakai T, Tamura T, Okada Y, Mikami H. Measurements of ligamentum flavum thickening at lumbar spine using MRI. Arch Orthop Trauma Surg. 2009;129(10):1415-9.

20. Kang YMS, Lee KS, Kim BH, Lee HS, Park KI, Lee SY. HM.; Moon,SH. Herniated Intervertebral Disk Induces Hypertrophy and Ossification of Ligamentum Flavum. J Spinal Disord Tech. 2014;27(7):382-9.

21. Hayashi K, Suzuki A, Abdullah Ahmadi S, et al. Mechanical stress induces elastic fibre disruption and cartilage matrix increase in ligamentum flavum. Sci Rep. 2017;7(1):13092.

22. Young-Mi Kang K-SS, Lee BH, Kim H-S, Lee K-I, Park S-Y. Hwan-Mo Lee, and Seong-Hwan Moon. Herniated Intervertebral Disk Induces Hypertrophy and Ossification of Ligamentum Flavum. J Spinal Disord Tech. 2014;27(7):382-9.

23. Zarate-Kalfopulos B, Reyes-Tarrago F, Navarro-Aceves LA, et al. Characteristics of Spinopelvic Sagittal Alignment in Lumbar Degenerative Disease. World Neurosurg. 2019;126:e417-e21.

24. Urrutia J, Besa P, Lobos D, et al. Lumbar paraspinal muscle fat infiltration is independently associated with sex, age, and inter-vertebral disc degeneration in symptomatic patients. Skeletal Radiol. 2018;47(7):955-61.

25. Lee SH, Park SW, Kim YB, Nam TK, Lee YS. The fatty degeneration of lumbar paraspinal muscles on computed tomography scan according to age and disc level. Spine J. 2017;17(1):81-7.

26. Jang TW, Ahn YS, Byun J, et al. Lumbar intervertebral disc degeneration and related factors in Korean firefighters. BMJ Open. 2016;6(6):e011587.

27. Ergun $\mathrm{T}$, Lakadamyali $\mathrm{H}$. The relationship between the prevalance and size of lumbar ossified ligamentum flavum and the presence and degree of facet joint degeneration. Eur J Radiol. 2012;81(11):3456-60.

28. Gellhorn AC, Katz JN, Suri P. Osteoarthritis of the spine: the facet joints. Nat Rev Rheumatol. 2013;9(4):216-24.

29. Panjabi MMO, Kifune TR, Arand M, Wen M, Chen L. A. Validity of the Three-Column Theory of Thoracolumbar Fractures. Spine (Phila Pa 1976). 1995;20(10):1122-7.

30. Dahlqvist JR, Vissing CR, Hedermann G, Thomsen C, Vissing J. Fat Replacement of Paraspinal Muscles with Aging in Healthy Adults. Med Sci Sports Exerc. 2017;49(3):595-601.

31. Bogduk N. Functional anatomy of the spine. Handb Clin Neurol. 2016;136:675-88. 
32. Wu CC, Jin HM, Yan YZ, et al. Biomechanical Role of the Thoracolumbar Ligaments of the Posterior Ligamentous Complex: A Finite Element Study. World Neurosurg. 2018;112:e125-e33.

33. Jun HS, Kim JH, Ahn JH, et al. T1 slope and degenerative cervical spondylolisthesis. Spine (Phila Pa 1976). 2015;40(4):E220-6.

34. lizuka Y, lizuka H, Mieda T, Tajika T, Yamamoto A, Takagishi K. Epidemiology and associated radiographic spinopelvic parameters of symptomatic degenerative lumbar scoliosis: are radiographic spinopelvic parameters associated with the presence of symptoms or decreased quality of life in degenerative lumbar scoliosis? Eur Spine J. 2016;25(8):2514-9.

\section{Figures}

Assessed for eligibility $(n=1088)$

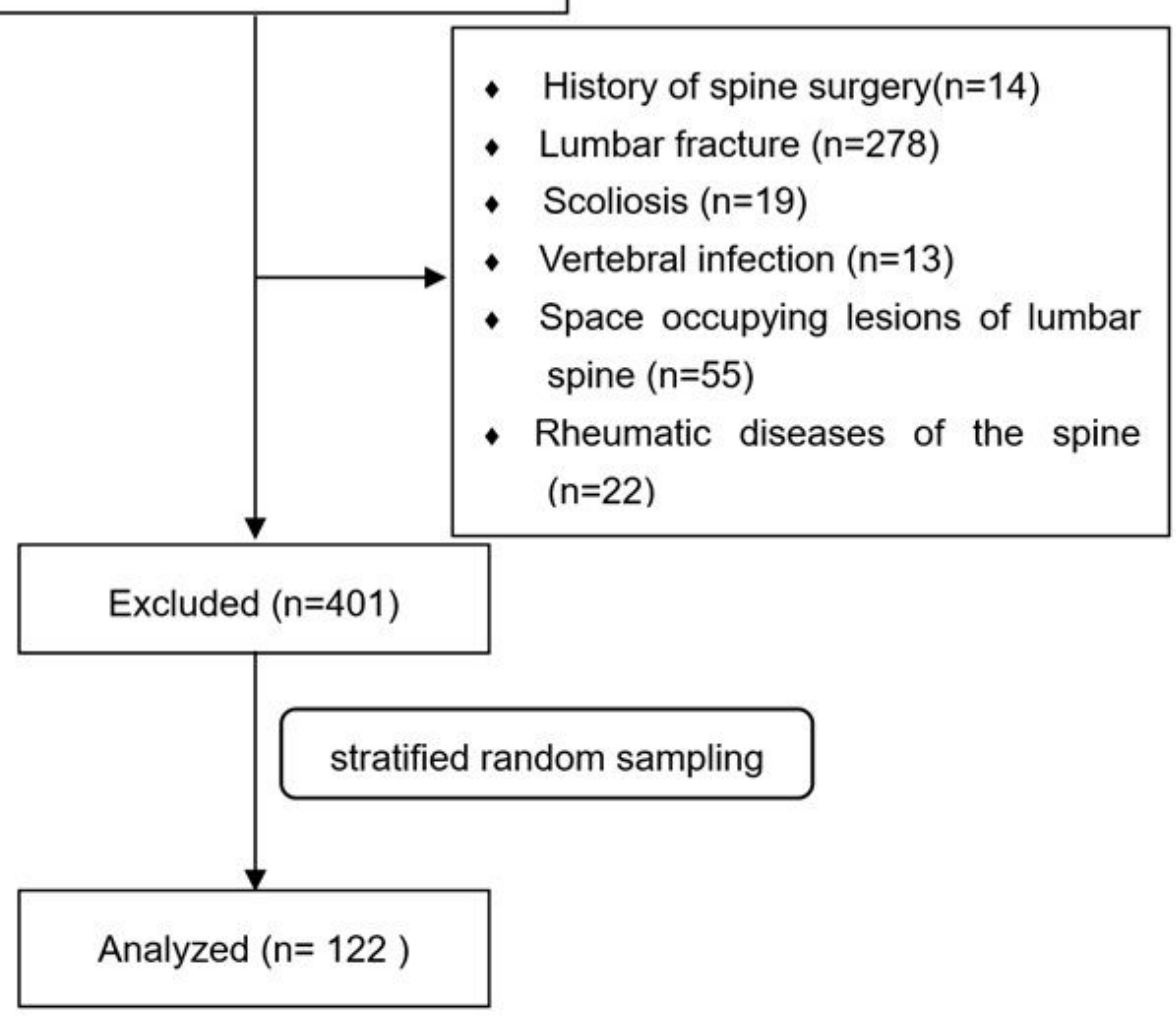

Figure 1

Flow diagram showing participant screening, exclusion, and analysis. 

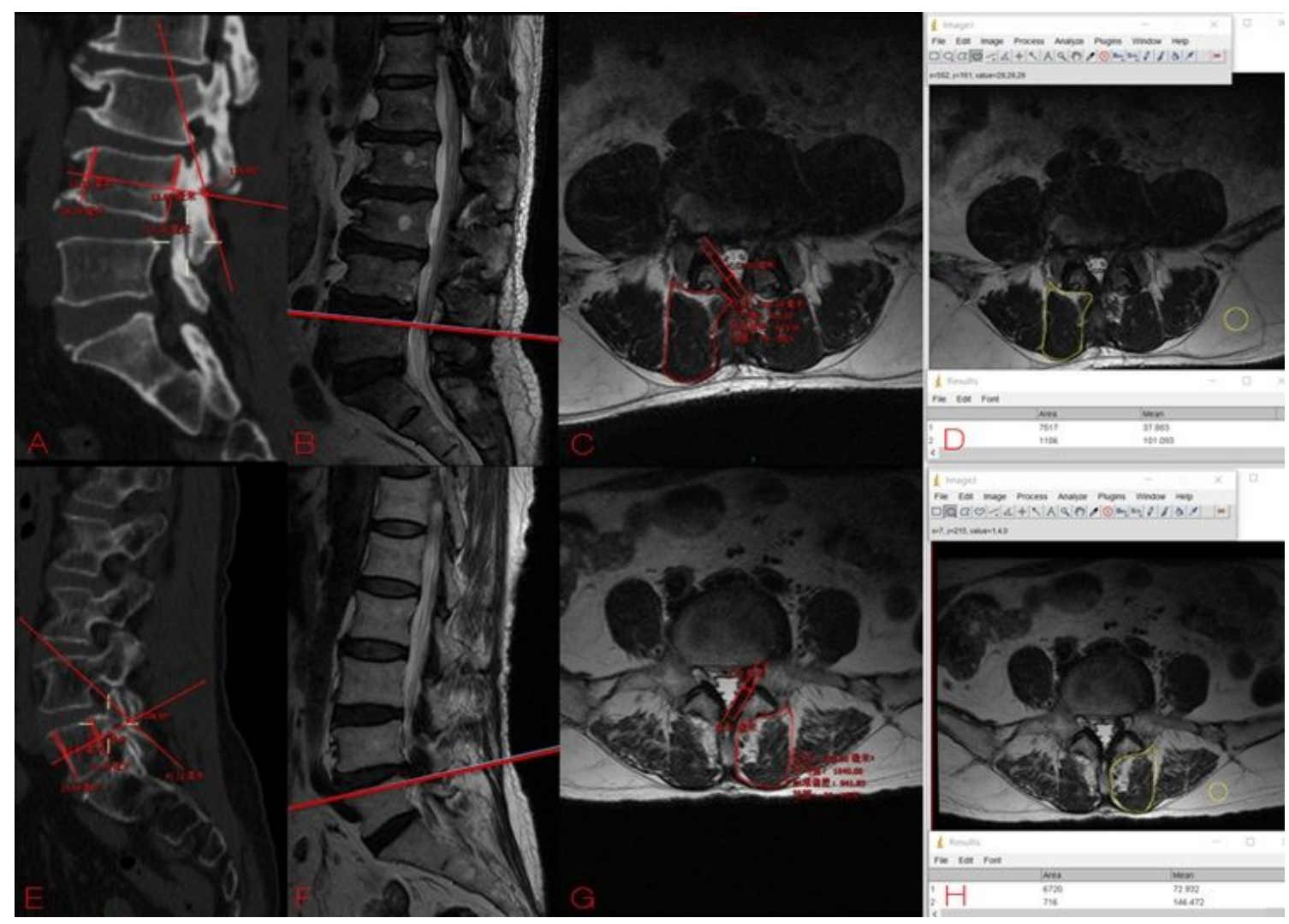

\section{Figure 2}

(A-D) are from a 64-year-old male patient, while (E-H) are from a 66-year-old female patient. (A) shows the LSA of L4, while (E) shows the LSA of $L 5$ on a sagittal CT image. (B, F) was chosen as the sagittal MRI image with which to analyze disc degeneration. (C) shows the right CSAof the multifidus, $(G)$ shows theleft CSA. $(D, H)$ shows the leftandrightmuscle-fatindex, as derived by Image J software.

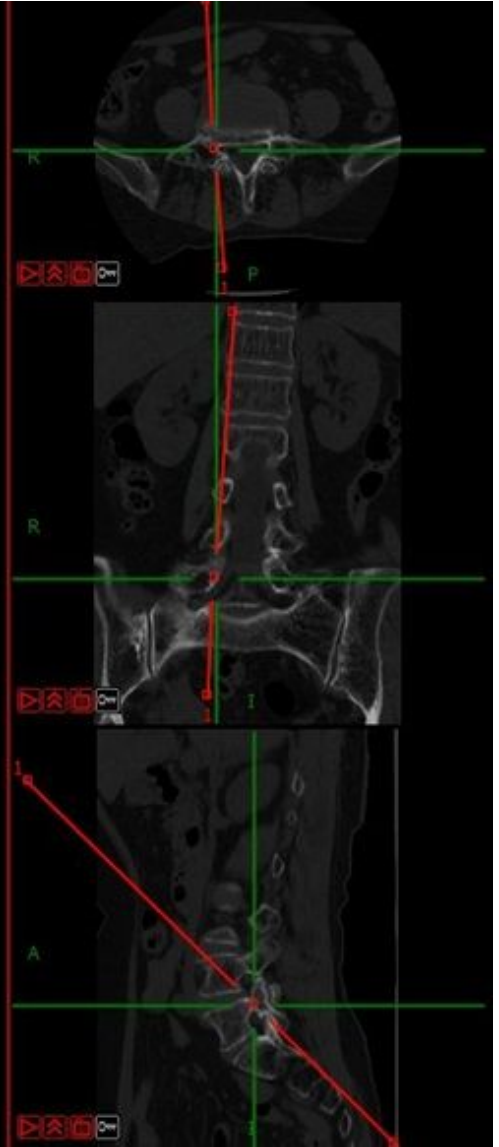


Figure 3

The proofreading procedure for measuring the lamina slope angle on a sagittal image from three-dimensional computerized tomography.

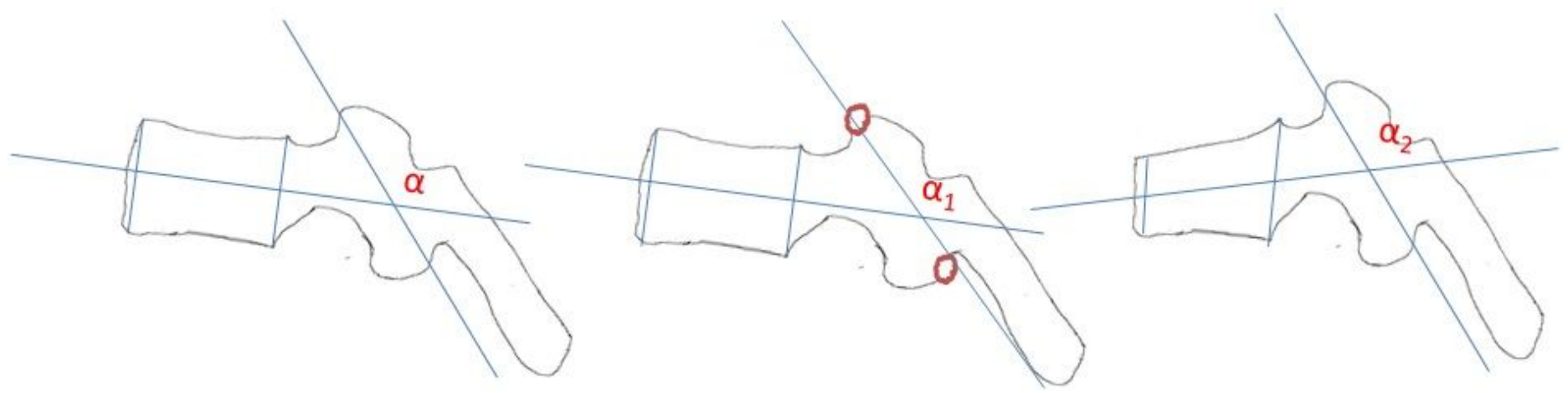

Figure 4

awas the lamina slope angle. a1 represented the bigger LSA with facet joint osteoproliferation. a2 represented the smaller LSA with compression fracture.

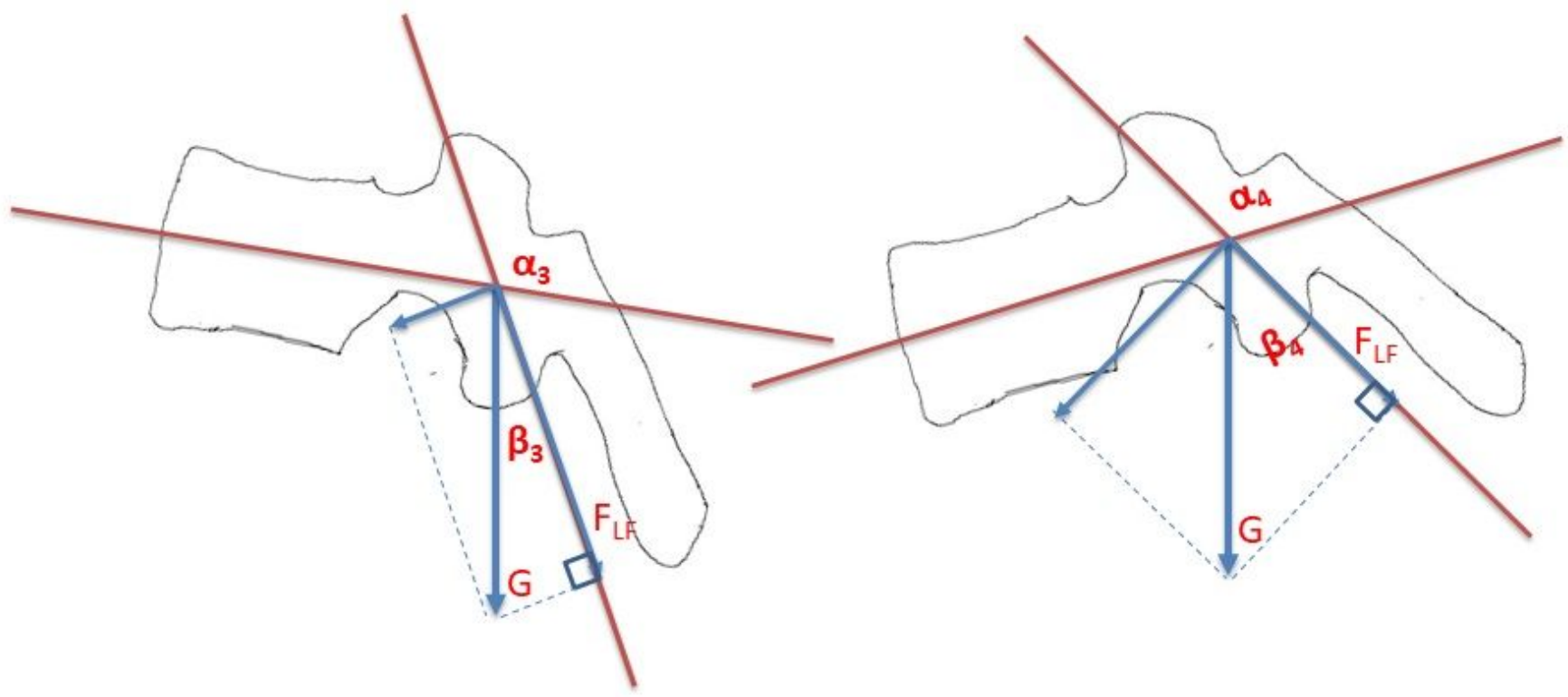

\section{Figure 5}

a3 represents the LSA of L4, while a4 represents the LSA of L5. This photograph shows the force analysis for the ligamentum flavum beneath the lamina. ' $G$ ' represents the gravity of our upper body, while 'FLF' represents the internal tension of LF. 